\title{
Opiniones de la calidad de vida desde la perspectiva de la mujer con discapacidad
}

\author{
María Victoria Martín- Cilleros, María Cruz Sánchez-Gómez, Sonia Verdugo-Castro y \\ Miguel Ángel Verdugo Alonso \\ viquimc@usal.es; mcsago@usal.es \\ Universidad de Salamanca, INICO (Instituto de Integración en la Comunidad) Avenida de la Merced 109, \\ 37005, Salamanca, España.
}

DOI: $10.17013 /$ risti.38.64-78

\begin{abstract}
Resumen: La calidad de vida se ve afectada ante una discapacidad, más aún cuando quien la presenta es una mujer. De cara a emprender futuras acciones se realiza un estudio dirigido a conocer la percepción que dicho colectivo tiene sobre su calidad de vida. Para ello se elaboró un cuestionario cualitativo. De las respuestas de 526 mujeres se realizó un análisis de los datos utilizando el software webQDA. Se observa que todas las dimensiones de calidad de vida se consideran afectadas en mayor o menor grado, siendo una variable a tener en cuenta el tipo de discapacidad; sin embargo, la percepción de falta de autonomía e inclusión es una constante en todo el colectivo. Los resultados indican la necesidad de continuar trabajando la independencia y autonomía a través de los apoyos, los cuales además de materiales o de recursos humanos deben ser emocionales desde el inicio de la discapacidad.
\end{abstract}

Palabras-clave: Percepción; Calidad de vida, Discapacidad; Mujer; Estudio cualitativo

\section{Views on quality of life as perceived by women with disabilities}

\begin{abstract}
The quality of life is affected by a disability, even more so when the person presenting it is a woman. With a view to undertaking future actions, a study is carried out aimed at knowing the perception that this group has about their quality of life. For this, a qualitative questionnaire was developed. From the responses of 526 women, data analysis was performed using webQDA software. It is observed that all the dimensions of quality of life are considered affected to a greater or lesser degree, is a variable to take into account the type of disability; however, the perception of lack of autonomy and inclusion is a constant throughout the collective. The results indicate the need to continue working on independence and autonomy through supports, which in addition to materials or human resources must be emotional from the beginning of the disability.
\end{abstract}

Keywords: Perception; Quality of life, Disability; Women; Qualitative study. 


\section{Introducción}

La observación presentada por el Comité sobre los derechos de las Personas con discapacidad acerca de las mujeres y las niñas con esta condición (Comité de Naciones Unidas sobre los derechos de las personas con discapacidad, 2016), indica que existen pruebas sólidas que demuestran que este colectivo afronta obstáculos en la mayor parte de los ámbitos de vida y que dichos obstáculos tienen que ver con: la igualdad de acceso a la educación, las oportunidades económicas, la interacción social y la justicia, el igual reconocimiento como persona ante la ley y la capacidad de participar en la política y ejercer control sobre sus propias vidas en diversos contextos como el de atención a la salud, incluidos los servicios de salud sexual y reproductiva, y de decisión sobre dónde y con quién desean vivir. Destaca así que las mujeres y las niñas con discapacidad tienen más probabilidad de ser objeto de discriminación que los hombres y los niños con discapacidad y que las mujeres y las niñas sin discapacidad.

El Comité señala la vivencia de una situación de invisibilidad que se ha perpetuado en situaciones de formas múltiples e intersectoriales de discriminación contra las mujeres y las niñas con discapacidad que son a menudo objeto de discriminación por motivos de género y/o discapacidad y también por otros motivos. Entre estos motivos, se alude a la edad, la discapacidad, el origen étnico, nacional o social, la identidad de género, la opinión política o de otra índole, la raza, la condición de migrante, refugiado o solicitante de asilo, la religión, el sexo y la orientación sexual. De este modo, indica que las mujeres con discapacidad pueden encontrarse en situaciones en las que varios motivos o factores, como el sexo y la discapacidad, entre otros o junto con otros, interactúen al mismo tiempo, de forma que son inseparables y conducen a situaciones de discriminación complejas agravadas y específicas. Destaca entonces que las mujeres con discapacidad son un grupo diverso en base a todas las variables anteriores y otras posibles, y advierte sobre la necesidad de tener esto en cuenta, pues en una mujer concreta la interacción de diversas de las variables descritas puede llevar a situaciones de discriminación aumentadas en base a tal conjunto complejo de interacciones (Comité de Naciones Unidas sobre los derechos de las personas con discapacidad, 2018).

Varias voces sugieren que el hecho de que las mujeres con discapacidad superen a los hombres, sobre todo a medida que la edad aumenta, no deriva solo de la aparición de condiciones biológicas, sino más bien de la existencia de estas situaciones y experiencias de discriminación relacionadas con la imposibilidad de tomar decisiones sobre sus propias vidas, el paternalismo con el que conviven, la falta de información y la falta de autonomía en la toma de decisiones en su vida cotidiana (Gomiz, 2017).

Como se ha comentado previamente, la historia de las mujeres con discapacidad es también una historia de resiliencia. Ciertamente, a pesar de la existencia de situaciones discriminación, exclusión, ausencia de visibilidad, vulneración de derechos y violencia contra ellas, muchas de estas mujeres ya están organizadas en movimientos activos y decididas a luchar para defender sus derechos. Del trabajo realizado por estos colectivos de mujeres han surgido muchas propuestas y planteamientos para avanzar en la mejora de sus condiciones de vida los cuales se detallan, entre otros, en el Segundo Manifiesto de los Derechos de las Mujeres y Niñas con discapacidad de la Unión Europea 
(Comité de Mujeres del Foro Europeo de la discapacidad, 2011) o, a nivel nacional, en el Segundo Plan Integral de Acción de Mujeres con Discapacidad 2013-2016 (CERMI, Fundación CERMI Mujeres, 2013). Datos recientes proporcionados por el Observatorio Estatal de la Discapacidad (OED, 2018), indican que, según la Encuesta Nacional de Salud realizada en 2017, un 22,8\% de las personas residentes en España se hab visto limitadas en los últimos seis meses para realizar las actividades de la vida cotidiana debido a un problema de salud. De nuevo, el porcentaje crece con la edad y es sustancialmente más alto entre las mujeres que entre los hombres.

En España, en el año 2008, según la Encuesta de Discapacidad, Autonomía Personal y Situaciones de Dependencia, EDAD, presentaban discapacidad más de tres millones y medio de personas. Casi el 60\% de estas eran mujeres y los porcentajes de mujeres con discapacidad con respecto a los hombres se veían incrementados a medida que aumentaba la edad (INE, 2009). En Castilla y León, en el año, en 2015 había un total de 169.637 personas con discapacidad, lo cual supone una prevalencia del 6,86\% sobre el total de la población. El 48,2\% de estas personas eran mujeres y el 51,8\% varones. El perfil es similar al reflejado en los datos mundiales y españoles afectando sobre todo a las personas de entre 50 y 89 años (que suponen el 69\% de las personas con discapacidad de Castilla y León). A partir de los 65 años y sobre todo de los 80 se produce también en Castilla y León una feminización de la vejez y las mujeres con discapacidad superan a los hombres. En concreto, para las discapacidades que generan mayor dependencia el número de mujeres duplica al de hombres. Finalmente, casi la mitad $(44,4 \%)$ de estas personas con discapacidad viven en entornos rurales con baja densidad de población o incluso despoblación lo que plantea la necesidad de crear prestaciones y recursos adecuados a las demandas concretas de personas con discapacidad que viven en zonas rurales dispersas (Consejería de familia e igualdad de oportunidades de Castilla y León, 2017).

Se considera de especial necesidad conocer la situación real de las mujeres con discapacidad al objeto de sensibilizar a la población y tener como base los resultados para emprender futuras acciones y acercarse a la situación de dicho colectivo.

\section{Método}

\subsection{Objetivos}

A través de una revisión amplia de bibliografía relacionada con el tema, se constata que no existen en la actualidad muchas investigaciones que recojan información sobre este colectivo, por lo que menos aún en Castilla y León. De ahí, la importancia de los resultados de este estudio, cuyo objetivo principal es conocer la percepción de la mujer con discapacidad en dicha Comunidad Autónoma teniendo en cuenta aquellos aspectos que contribuyen a su desarrollo integral e inclusivo. Este estudio es de especial interés, puesto que supone analizar las demandas de las mujeres con discapacidad y proporcionar sugerencias de mejora del sistema de dependencia (Martín-Cilleros, Sánchez-Gómez, Verdugo-Alonso, Crespo-Cuadrado, Sánchez-García, Caballo-Escribano, VerdugoCastro y Manjón-García, 2020) 


\subsection{Participantes}

El colectivo al que estuvo dirigida la investigación fue la mujer con discapacidad cuya residencia se ubicara en la comunidad española de Castilla y León. Dicha comunidad está formada por ocho provincias Para acceder a las mujeres con discapacidad se recurrió a instituciones oficiales y asociaciones de personas con discapacidad. Además, se enviaron cartas de solicitud de participación a los Centros de Acción Social de las zonas rurales de la Comunidad de Castilla y León, solicitando su colaboración en el estudio para que nos permitan llegar a las mujeres con discapacidad de estas zonas, para las personas con discapacidad auditiva se contó en el estudio con una traductora de lengua de signos. A través de la información proporcionada se seleccionó una muestra representativa y aleatoria de la población de la zona rural y urbana de la Comunidad bajo los siguientes criterios de inclusión:

- Mujer con algún tipo de discapacidad, o discapacidad múltiple. En el caso que la discapacidad fuera intelectual, la persona debería tener las habilidades de comprensión adecuadas para poder responder al cuestionario.

- Con una edad superior o igual a los 18 años.

- Residente en cualquier municipio de la Comunidad de Castilla y León

\subsection{Procedimiento}

En situaciones como las planteadas en este estudio, en que los contextos a investigar están claramente definidos (mujeres con discapacidad de Castilla y León), y la población implicada en la intervención es limitada, un planteamiento meramente extensivo no permitiría la realización de un análisis minucioso como el que propone el enfoque intensivo (Sánchez-Gómez, Martín-Cilleros, Costa y García-Peñalvo, 2018). Por estas razones teórico-metodológicas y pragmáticas, se consideró pertinente la utilización de métodos cualitativos para identificar y proponer líneas de intervención.

Para el estudio se elaboró un cuestionario ad hoc de preguntas abiertas dirigido a conocer expectativas, creencias, percepciones y opiniones de las mujeres con discapacidad castellano-leonesas. El cuestionario, diseñado con un lenguaje inclusivo y siguiendo los criterios de accesibilidad, se subió a la plataforma SurveyMonkey, creando un enlace al que pudieron acceder todas las personas que desearon cumplimentar la encuesta. La difusión se realizó a través de representantes, a instituciones oficiales y asociaciones de personas con discapacidad. Previo a la recolección de datos se elaboró igualmente un consentimiento informado de acuerdo con la ley de protección de datos.

Se realizó un muestro incidental de la población de la zona rural y urbana de la Comunidad de Castilla y León, con respuesta de 526 personas, ofreciendo la información las propias interesadas, cuidadores primarios o tutores.

Para el estudio, el foco de interés ha recaído sobre las ocho dimensiones de la calidad de vida propuestas por Schalock y Verdugo (2007). El análisis de los discursos se ha realizado en primer lugar, a través de una transcripción del contenido y con confidencialidad sobre los datos personales se ha aplicado el análisis. Los análisis de contenido clásico de las respuestas abiertas de dicho cuestionario se realizaron siguiendo las fases: reducción, disposición y transformación de los datos (Sánchez-Gómez y Martín 
Cilleros, 2017; Sánchez-Gómez, Palacios Vicario, y Martín García, 2015), utilizando el software de análisis cualitativo webQDA. Se trata de un software en línea, que fomenta el trabajo colaborativo por medio de proyectos compartidos. Además, trabajando colaborativamente se puede trabajar síncrona y asíncronamente. Dicho software permite la importación de fuentes internas y externas, la generación de categorías y codificación del contenido, y el análisis de este a partir de la gestión, filtro y búsqueda de elementos (palabras más frecuentes, búsqueda de texto, etc.) (Costa, 2016; Reis y Costa, 2017; Souza et al., 2011). Para el presente estudio se ha utilizado la búsqueda de texto. Esta opción reporta al investigador una útil herramienta sobre el contenido asociado a ese término, en las diferentes fuentes, preguntas planteadas y categorías descritas. Otra herramienta utilizada es la búsqueda de palabras más frecuentes, de las cuales se podría extraer nubes de palabras. Y finalmente, se puede presentar la información del sistema categorial en un árbol de códigos.

En la Figura 1 se presenta el dashboard del panel de inicio de un proyecto en webQDA.

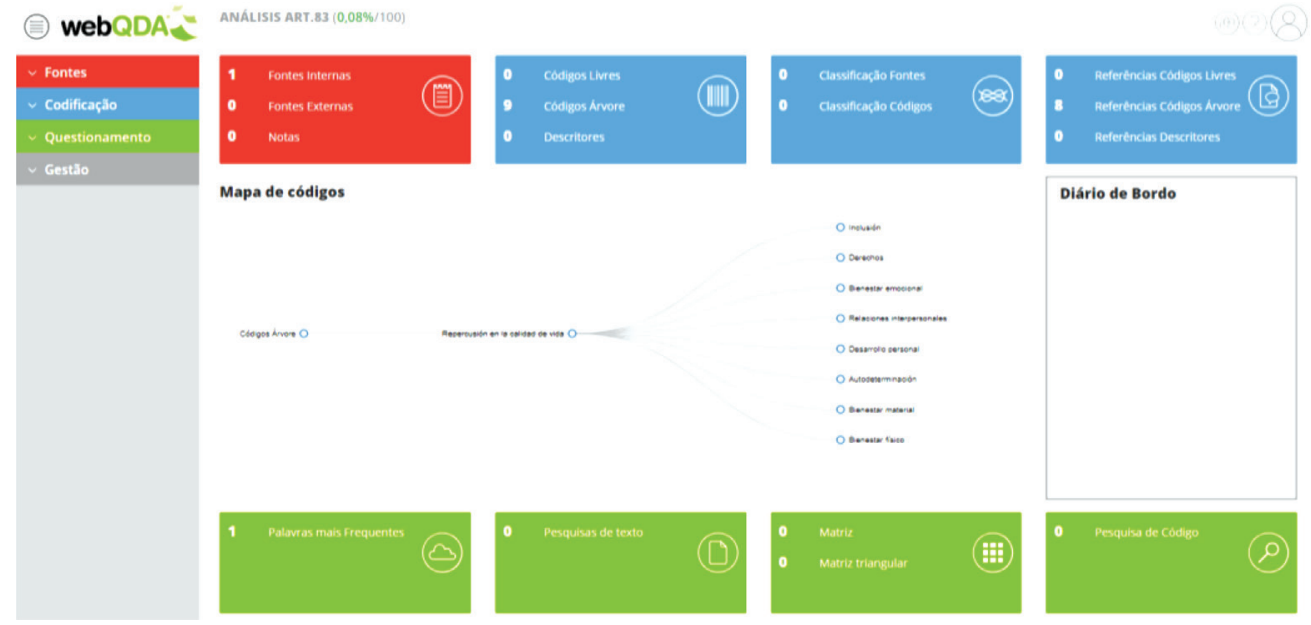

Figura 1 - Dashboard de webQDA. Fuente: webQDA

Siguiendo los criterios para garantizar la calidad en la investigación cualitativa (Palacios, Gutiérrez y Sánchez 2013), la codificación fue realizada bajo la supervisión del equipo, para así poder garantizar la credibilidad del proceso.

\section{Resultados}

La presentación de resultados se efectuará siguiendo la siguiente estructura:

1. Presentación del árbol de códigos y mapa de densidad, consta de categorías que reflejan la presencia y estructura de las percepciones y experiencias respecto a las dimensiones de calidad de vida. Esta disposición representa la denominada "teorización o generación de teoría” concebida a partir de las expresiones de los 
participantes.

2. Análisis semántico: frecuencia de ocurrencia de las palabras y búsqueda de términos relevantes

\subsection{Representación árbol de códigos}

Previo al proceso de categorización, se realizó un análisis global que consistió en reuniones del equipo para clarificar conceptos clave. En nuestro caso la calidad de vida de las mujeres con discapacidad. Antes de la codificación se leyó varias veces el texto para detectar afirmaciones centrales, anotar las ideas y palabras clave; y, sus posibles definiciones. El proceso se completó realizando juicios de valor sobre este análisis, lo que permitió elaborar un libro de códigos para que los codificadores no cometieran errores al asignar texto a una categoría correspondiente (Martín-Cilleros et. al, 2020).

El mapa conceptual obtenido está centrado en la calidad de vida (CV), a partir de la cual se distinguen las subcategorías correspondientes a las ocho dimensiones propuestas por Schalock y Verdugo (2007), son las que se presentan en el siguiente mapa conceptual (figura 2):

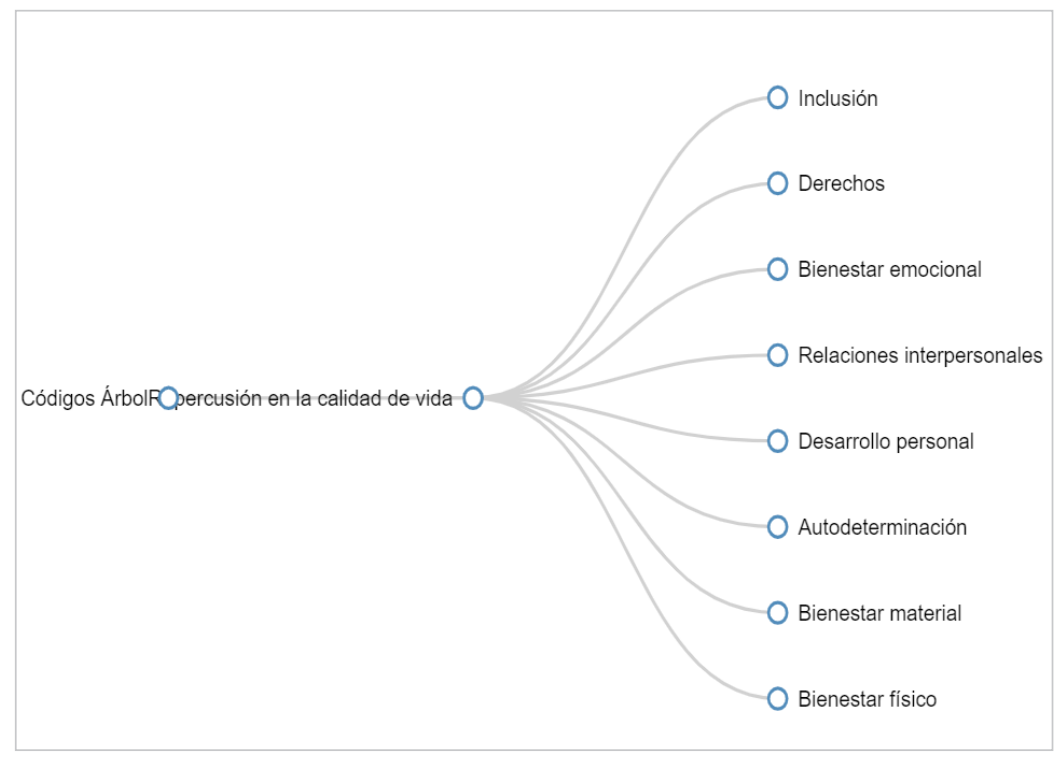

Figura 2 - Árbol de códigos. Fuente: webQDA

En el siguiente mapa de densidad (figura 3), pueden observarse las subcategorías realizadas para cada dimensión de calidad de vida. Este modelo visual dinámico que ofrece el software webQDA permite acceder al número de referencias que hay en la categoría. En general, las dimensiones más comentadas hacen referencia a la autodeterminación e inclusión, siendo los temas menos abordados las relaciones interpersonales y el bienestar físico. 


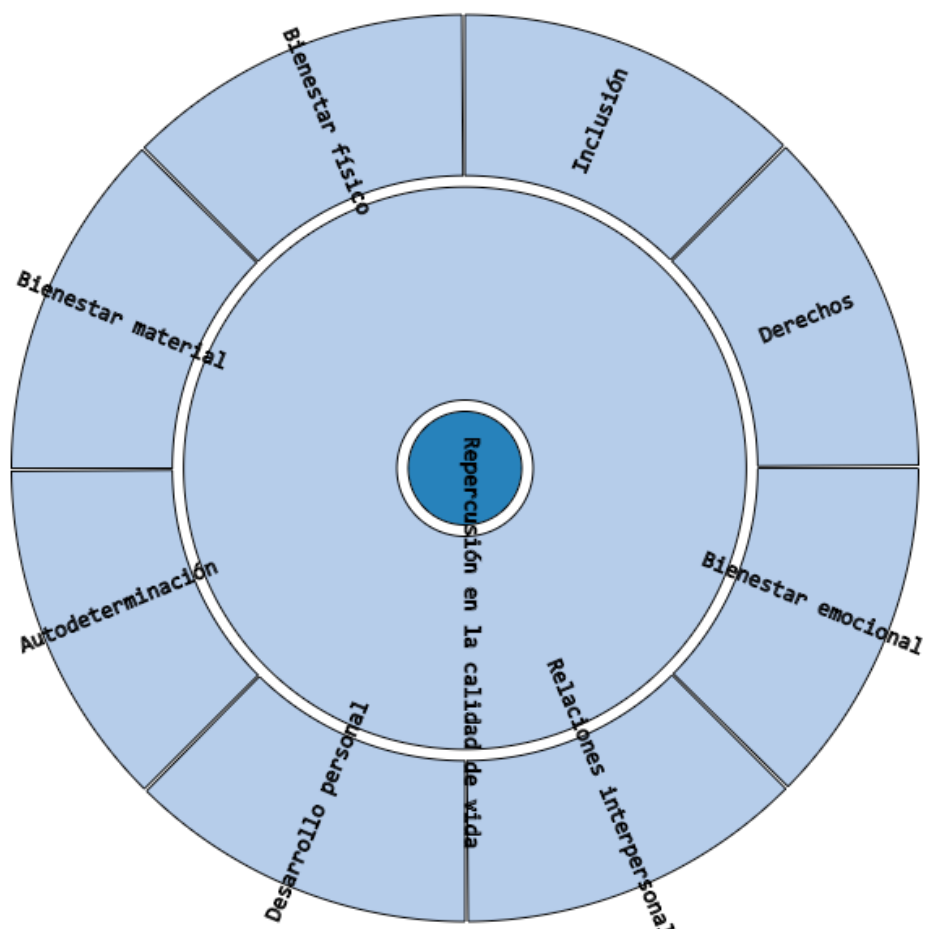

Figura 3 - Mapa de densidad. Fuente: webQDA

La autodeterminación se encuentra limitada en diferentes aspectos como puede observarse en sus opiniones "que mi tutor casi siempre decide por mí y no me gusta esta situación.”, “que no me gustan que decidan por mí, me considero divertida e independiente y no me dejan ser como yo quiero. me gustaría que me dejaran estar con mis amigos y pareja más libremente".

De los comentarios vertidos en esta subcategoría, aproximadamente un $40 \%$ fueron a cargo de personas que presentaban discapacidad múltiple, donde reflejan experiencias relacionadas con la total dependencia de los padres "dependo totalmente e mis padres", seguido de un $24 \%$ por personas con discapacidad intelectual, donde los comentarios hacen referencia a no poder elegir en diferentes aspectos de sus vida "no he elegido dónde ni con quién vivir ", "no me dejan ir a conciertos “, "no siempre se respetan mis decisiones"; un $15 \%$ son personas con problemas de salud mental que abarcan los dos aspectos comentados anteriormente con manifestaciones del tipo "no se administrar el dinero. me tienen que ayudar y a veces gasto todo lo que tengo en objetos que no necesito", "en cuanto a la relación de familia a veces dicen que soy caprichosa que tiene que ser lo que yo diga", "tener derecho a la autodeterminación, tomar nuestras propias decisiones"; y un 12\% aquellas que presentan parálisis cerebral que vuelven a enfocarse 
más en la total dependencia que tienen de un apoyo "total y absoluta, siempre me han ordenado lo que tenía que hacer, cuándo y de qué manera", "constante vigilancia".

Esta dimensión fue menos tenida en cuenta por las personas con alguna discapacidad física o enfermedad rara.

En cuanto a la segunda dimensión más tratada, la inclusión, cerca de un $29 \%$ de los comentarios proceden de personas con discapacidad múltiple, donde se manifiestan aún barreras arquitectónicas "sobre todo las barreras arquitectónicas pues voy en silla de ruedas y a veces no puedo acceder a ciertos sitios, piscinas, cines", "mi enfermedad no me permite ciertos accesos", "porque hay barreras" y en algunas circunstancias la persona se ha desplazado para poder participar más en la comunidad "dentro del pueblo nos hemos ido a vivir más al centro a casa de mi padre a fin de que él estuviese más integrado en el entorno. no tal aislado como en nuestro anterior domicilio"; seguido de $15 \%$ con comentarios ofrecidos por personas con discapacidad intelectual donde las barreras siguen siendo aún mentales "al tener una discapacidad muchas empresas cuando ven tu curricular te juzgan sin conocerte y prefieren no contar contigo", "cuando las personas se enteran de que tengo una discapacidad me discriminan"; al igual que ocurre con las personas con problemas de salud mental "al tener una discapacidad no visible se meten contigo si no cedes el asiento en el autobús o no contestas rápidamente a sus preguntas", "la gente me mira como si fuese un bicho raro, sobre todo en la familia de mi padre hemos tenido exclusiones de celebraciones familiares". En esta dimensión cabe destacar la ausencia de comentarios por personas con TEA y un escaso 3\% de participación por personas con discapacidad auditiva, visual, daño cerebral o enfermedades raras.

Con relación a la subcategoría de bienestar emocional, su contenido fue mencionado entre un 17-20\% por las personas con multidiscapacidad y discapacidad física, seguido de personas con problemas de salud mental y discapacidad intelectual. Al igual que en la dimensión de inclusión las personas que menos comentarios realizaron fueron la discapacidad auditiva, visual, enfermedades raras y TEA que no comentaron nada. Lo característico de todas las discapacidades en esta dimensión es la presencia de sentimientos de depresión "en ocasiones estoy deprimida, sobre todo en verano", "donde no haga mucho calor para ponerme un bañador de cuello vuelto para el linfedema y por supuesto que no se me vea el cuerpo escombro que me ha quedado" (mujer con multidiscapacidad), "tuve que acudir al psiquiatra " (mujer con discapacidad física), "he perdido memoria y he empezado a entrar en depresión" (mujer con problemas de salud mental), "en mi casa tengo un "cartel" que pone enferma mental. percibo que mi enfermedad condiciona a mi familia a la hora de contar conmigo en muchas cosas.", "a veces me siento triste y deprimida y no sé a quién acudir" (mujer con discapacidad intelectual), "inseguridad en el trato con los demás" (mujer con discapacidad visual), "estoy algo frustrada" (mujer con discapacidad auditiva).

El desarrollo personal, como subcategoría, es el tema que ha ocupado el $45 \%$ de los comentarios realizados. Este tema ha sido más abordado por las personas con multidiscapacidad, formando el $43 \%$ de todos los comentarios en dicha dimensión indicando sus limitaciones derivadas de su discapacidad "mis limitaciones han hecho un cambio radical de mi vida", "no puedo hacer casi nada de lo que me gusta por uno u 
otro problema de salud", "no poder realizar tareas cotidianas", "mis aficiones han tenido que cambiar la lectura me apasiona pero ya no puedo leer", "no pude seguir estudiando y nunca he tenido trabajo", "limito seguir estudiando"; seguido de las personas con discapacidad intelectual que ofrecen alrededor de un $11 \%$ de comentarios relacionados con el desarrollo personal en el ámbito académico como "menos formación por discapacidad", "no terminar estudios y buscar trabajo", "estudias mas"; seguido de las personas con problemas de salud mental con un 7,4\% del discurso, que hacen una mayor referencia a su limitación en el desarrollo profesional "me ha afectado sobre todo a nivel laboral porque la gente huye de una persona depresiva”, "al tener enfermedad mental veo que no puedo soportar el estrés como antes"; y discapacidad física con el mismo porcentaje anterior donde manifiestan igualmente la misma limitación "no siempre los trabajos están adaptados", "al dejar de hacer mi trabajo".

De los 69 comentarios realizados sobre bienestar material, aproximadamente el 17\% ha sido a cargo de las personas con discapacidad múltiple donde hacen referencia a modificaciones que han tenido que realizar y les ha supuesto un gasto económico "Dafos y cama articulada", "nos hemos ido a vivir de la ciudad a un pueblo porque es más fácil la vida", "hemos tenido que adaptar el cuarto de baño tengo que tener los armarios a mi alcance". Alrededor del $12 \%$ de los comentarios han sido ofrecido por las personas con discapacidad física, las cuales expresan los mismos motivos que las mujeres anteriores "debo tener plataformas de acceso especial, ya que no puedo subir ni bajar escaleras", junto con gastos económicos derivados de tratamientos sanitarios "costearme los tratamientos re-habilitadores", "gaste todo el paro durante la enfermedad, y a los 20 días de terminar con la radioterapia, un mes y medio de la quimio un médico dijo que ya estaba bien de chupar tanto del bote y me dio el alta...”. Un $8 \%$ de personas con discapacidad intelectual manifestaron en esta dimensión los escasos ingresos que tienen "el no tener ingresos me ha dificultado poder participar de cosas que me gustan", "el dinero del que dispongo es escaso".

En relación a la dimensión de derechos, al igual que en las anteriores las personas con múltiples discapacidades, son las que más comentarios generan, indicandola importancia de cumplir con los derechos de las personas con discapacidad "derecho a un empleo digno , tener derecho a la autodeterminación, tomar nuestras propias decisiones", "Falta de ayuda y apoyo", "hemos conseguido cosas en cuanto a derechos pero falta mucho por conseguir,, el acceso a los servicios públicos a veces es complicado”. Sin embargo, las personas con discapacidad en esta dimensión reivindican el derecho a un trabajo, o un trabajo mejor "Poder cambiar a otro trabajo", "No he tenido la posibilidad de tener un trabajo y de ganarme un dinero de forma independiente", "Me gustaría trabajar". En las situaciones de las personas con discapacidad física, estos derechos se tuvieron, pero su condición hizo que se vieran mermados, como nos indican comentarios como "En la empresa en la que estaba, cuando dije que tenía cáncer me despidieron, y como premio a su maravillosa conducta, la juez, dijo que era una perfecta decisión", "Nadie exige que digas que discapacidad tienes, pero si no dices cuál es, olvídate de ser contratada”, "Despido por no cambiarme puesto de trabajo".

La dimensión de bienestar físico ha sido más abordada por las mujeres con multidiscapacidad, discapacidad física, salud mental y discapacidad intelectual (con un 
44\%, 30\%, 14\% y 8\% respectivamente). En el caso de las discapacidades múltiples los comentarios hacen referencia a sus problemas de salud y la necesidad de medicación para su estado "debo cuidar mucho mis defensas la mínima infección perjudica mi enfermedad", "mucho por el dolor y cansancio extremo con nada que hago", "he cogido mucho peso, además tengo artritis y soriasis", "corticoides y antiepilépticos". Comentarios parecidos realizan las personas con discapacidad física "caídas al andar", "con cuidados por medicación”, "porque me encuentro muy cansada". En las mujeres con problemas de salud mental su malestar se debe a los efectos secundarios de la medicación "pierdes tu agilidad, las pastillas son beneficiosas para unas cosas, pero malas para otras", "sobrepeso, tabaquismo", "debido a la medicación ando más despacio y no aguanto un esfuerzo grande de tiempo prolongado".

Finalmente, en la dimensión de relaciones interpersonales las mujeres con discapacidad múltiple, que han expresado el $32 \%$ de los comentarios, hacen referencia a sus dificultades para iniciar la relación "la gente ve raro a personas jóvenes con tantos problemas de salud. eres una carga", "me cuesta mucho hablar con la gente", "me cuesta mucho relacionarme con la gente, hablo con cualquiera sin ver el peligro. no tengo un grupo de amigos o amigas".

Sin embargo, las personas con problemas de salud mental, que ofrecen el $22 \%$ de los comentarios, se quejan de la pérdida de relaciones "han surgido bastantes problemas", "ha limitado mis relaciones", "debido a la medicación y a la enfermedad me relaciono menos. a veces me encuentro mal y esto influye en mis relaciones.", "deje de salir con mis amigos y poco a poco he ido perdiendo relación con ellos". Las mujeres con discapacidad intelectual (16\% del contenido), en este aspecto indican la ausencia de relaciones e incluso una mayor vulnerabilidad a situaciones de abuso "porque tenía que ir sola, me gustaría tener amigos o amigas", "me ha afectado en la relación de pareja, a la hora de encontrar pareja y a la hora de mantener relaciones íntimas con mi pareja cuando la he tenido", "de no tener pareja", "en mi pueblo he vivido situaciones de falta de respeto por parte de otros. me han insultado y en ocasiones he tenido situaciones de abuso sexual por ser más vulnerable”.

Por su parte, las mujeres con discapacidad física (un 11\% del contenido) hacen más referencia a la pareja "hasta el extremo de las relaciones sexuales, duermo con CPA", "muchos problemas de relación entre la pareja"; al igual que a la pérdida de las amistades "los amigos te abandonan porque eres una carga", "mi entorno laboral y las amistades sin los dos ámbitos que más empeoraron, esto me ha caudado mucho sufrimiento y ha causado muy mala calidad de vida en general”. Las mujeres con parálisis cerebral (al igual que la anterior con $7 \%$ ) indican una ausencia de relaciones "falta de red social de apoyo ante circunstancias vitales complicadas", "me cuesta mucho relacionarme con mis compañeros, porque no se seguir sus conversaciones y a veces no me interesan porque no las entiendo, y no se seguir su ritmo entro tarde a responder y como ya dije antes dobles sentidos y no me gustan las bromas"

\subsection{Análisis semántico}

Las palabras más frecuentes en los comentarios ofrecidos por las participantes son las que se presentan en la siguiente figura (figura 4). 


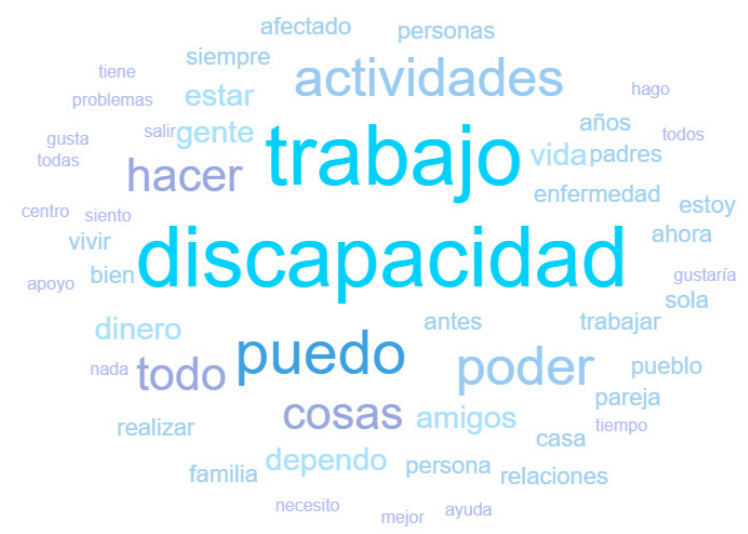

Figura 4 - Nube de palabras más frecuentes. Fuente: webQDA

Se observa como la palabra discapacidad es la más frecuente, siendo el eje de la calidad de vida de la persona "He tenido que adaptar muchas cosas pequeñas a mi discapacidad"; que además condiciona el acceso a un puesto trabajo, como nos indica el comentario "Es mucho más difícil cuando se tiene una discapacidad encontrar un trabajo. A mí me gustaría trabajar y encontrar un trabajo estable", "Al tener una discapacidad muchas empresas cuando ven tu currículum te juzgan sin conocerte y prefieren no contar contigo". Incluso con la aparición de la nueva condición han llegado a perder el puesto laboral como manifiesta alguna mujer "Tenía un puesto de trabajo fijo, y me quedé en el paro por no poderlo realizar debido a mi discapacidad", o tienen miedo de perderlo "En el trabajo no poder decir que enfermedad padeces por miedo a que no te renueven o perder el trabajo".

La pérdida de trabajo hace que se desestabilicen no solo el bienestar material, sino que influye en otras dimensiones de calidad de vida como la autodeterminación "No es fácil encontrar un trabajo y con ello medios para tener una vida autónoma e independiente". Esta autodeterminación se manifiesta en el discurso reflejada en términos como "puedo" o "poder", como nos indican los comentarios "No puedo desempeñar cualquier trabajo", "Yo no puedo vivir solo dependo de otra persona las 24 horas los 365 días del año, "no puedo elegir lo que quiero no puedo elegir donde vivir", "no poder vivir sola".

Las actividades de la misma manera se ven condicionadas por la discapacidad "no he podido hacer muchas actividades que me hubieran gustado", entre otras cosas por no tener las habilidades necesarias, o incluso por la falta de inclusión "Depende de cómo sean las personas que componen esos grupos o asociaciones". Para la realización de estas actividades, generalmente una gran mayoría, las mujeres necesitan de apoyo "necesito que alguien dirija mis actividades", "dependo de otras personas para muchas actividades", siendo principalmente familiares "dependo de mi marido", "dependo de mis padres", "dependo de otra persona las 24 horas los 365 días del año", "dependo de un familar".

Entre los términos más relevantes en el discurso se encuentra la palabra "dependo", muy relacionada con la necesidad de apoyos dirigidos a cubrir las necesidades básicas de la 
vida cotidiana, así como en el acompañamiento en los desplazamientos y apoyo escolar "Necesito apoyo de una tercera persona", "Dependo siempre de un apoyo para realizar las actividades anteriores". Entre los beneficios que manifiestan acerca de los apoyos, estos son considerados como imprescindibles para proporcionar más autonomía y descanso a los familiares, asistir a revisiones médicas, poder planificarse mejor, en general lo ven importante para todos los aspectos de sus vidas.

Igualmente se hace referencia en múltiples ocasiones a la necesidad de apoyos a nivel psicológico y emocional, debido a todas las circunstancias a las que deben enfrentarse "Si no tienes ningún apoyo, no puedes salir adelante en algunas situaciones, te vuelves mucho más vulnerable y puedes sufrir depresión...", "Falta de red social de apoyo ante circunstancias vitales complicadas”.

\section{Discusión}

Las apreciaciones expresadas por las mujeres indican que la calidad de vida de este colectivo se encuentra afectada en todas las dimensiones propuestas por Schalock y Verdugo (2007). Resaltan la falta de autonomía, con una constante sensación de dependencia, y falta de elección, debiendo adaptarse a sus cuidadores o tutores. Si bien hay una mayor presencia de personas con discapacidad en la comunidad, no disfrutan plenamente de ella siendo aún la participación una asignatura pendiente, bien por las barreras físicas o, por las más difíciles de eliminar, como son las mentales. Las redes sociales disminuyen, reduciéndose el círculo de amistades, y complicándose la creación de nuevas redes que no estén relacionadas con su nueva situación, donde juega un papel importante las dificultades para la participación en la comunidad, como se comentó anteriormente. Otras veces cuando la discapacidad aparece en la vida de la persona o la familia, van desapareciendo amistades, debido a que la condición limita las actividades que se hacían anteriormente y esto condiciona al grupo de amigos o amigas (MartínCilleros et al., 2020).

Del mismo modo se ve afectada la dimensión de desarrollo personal, viéndose reflejado en los comentarios la dificultad de encontrar trabajo debido a la condición en la que se encuentran, a la que se suma muchas veces la de ser mujer y con cierta edad. Incluso algunas de las participantes indican que la discapacidad ha derivado en una situación de desempleo. Aunque todas las dimensiones se encuentran vinculadas, en este caso el trabajo, como un aspecto incluido en el desarrollo personal, hace que el bienestar material vaya en detrimento, debido a un menor ingreso y un mayor gasto sanitario, arquitectónico o de otro tipo como es la contratación de uno o varios asistentes personales, necesarios por la situación de discapacidad. Nos encontramos ante un colectivo con serios problemas de salud, algunos provocados por la medicación, y a pesar de que la dimensión del bienestar físico no ha tenido una frecuencia muy numerosa de comentarios se observa que influye en la situación de dependencia que perciben (Martín-Cilleros et al., 2020).

Al igual que otros estudios han venido observando (Gomiz, 2017; Martín-Cilleros, Navarro-Yuste y Sánchez-Gómez, 2017; Shu y Conde, 2009), los comentarios indican la presencia de problemas de salud mental en las participantes, entre los que se encuentran problemas emocionales, como baja autoestima, que acaban derivando en depresión u otros problemas de salud mental más serios, momento en el cual comienzan a ponerse 
en manos de profesionales. A partir de estas manifestaciones se observa la necesidad de ofrecer un apoyo emocional desde los inicios de la discapacidad cuando esta surge en el desarrollo evolutivo de la persona, puesto que cuanto mayor es la edad de la mujer más se ve afectada su salud mental; así como insistir en la necesidad de la educación emocional desde las etapas infantiles, incidiendo más aún en aquellas personas que presentan una discapacidad desde el nacimiento.

Como se puede apreciar todas las dimensiones de calidad de vida se encuentran vinculadas y se observa como la urgencia de resolver sus necesidades básicas derivadas de su discapacidad con comentarios como "he tenido que cambiar mi lugar de residencia", hace que sus ingresos se vean mermados para poder realizar otras actividades de carácter lúdico, que unido a la dificultad de participación en la comunidad como se manifiesta en comentarios del tipo "uso silla de ruedas y no puedo caminar y ello me impide poder entrar en muchos sitios"; la progresiva disminución de su círculo de amistades "He reducido el círculo familiar y amistades", la dependencia de otras personas "no salgo apenas de casa, al no manejarme sola no me mantengo de pie, más de unos segundos, salgo a la calle con mi marido" y en algunos casos la progresiva percepción negativa de su autoimagen "hacen sentirme inferior", van minando la salud mental y repercutiendo en el bienestar emocional expresado por comentarios como "a veces me siento triste y deprimida", "estoy siempre de mal humor", "me emociono con facilidad y no lo puedo controlar". Como indican Shalock y Verdugo (2007) las dimensiones de calidad de vida presentan una jerarquía parecida a la de las necesidades humanas, donde el bienestar emocional sería el culmen final y donde es necesario tener cubiertas las dimensiones más básicas como son el bienestar físico, material y los derechos fundamentales para avanzar en el desarrollo personal y la autonomía, que permitan unas relaciones interpersonales ajustadas a la edad de desarrollo y una inclusión social.

\section{Conclusiones}

De los resultados se desprende la necesidad de continuar trabajando la independencia y autonomía, siendo la figura del asistente de apoyo personal como una pieza clave tanto para la persona con discapacidad, al permitirle mayor autonomía y margen de organización, como para los familiares que les sirve de descanso y la posibilidad de ejercer el auténtico rol de familiar y no tanto de cuidador. Actualmente esta figura solo se utiliza para cubrir las necesidades básicas de la vida diaria como vestirse, asearse o acompañamiento a centros médicos. Es necesaria una mayor disponibilidad de estos, tanto en número como en horas. Los comentarios indican la necesidad de más asistentes personales, regulados por la administración y con una formación determinada, hasta ahora sin regular. Igualmente, es necesaria la sensibilización de la población en general, facilitando más espacios de participación diseñados acorde a los principios de diseño universal (Martín-Cilleros et al., 2020) que permitan llegar a una mayor inclusión social.

\section{Referencias}

CERMI, Fundación CERMI Mujeres (2013). II Plan Integral de Acción de Mujeres con discapacidad 2013-2016. CINCA. 
Comité de Mujeres del Foro Europeo de la Discapacidad (2011). $2^{\circ}$ Manifiesto de los derechos de las Mujeres y Niñas con Discapacidad de la Unión Europea. Una herramienta para activistas y responsables políticos. EMAKUNDE-Instituto Vasco de la Mujer.

Comité sobre los derechos de las personas con discapacidad de Naciones Unidas (2016). Observación general número 3 sobre las mujeres y las niñas con discapacidad. Naciones Unidas: Convención sobre los derechos de las Personas con Discapacidad. http://www.fundacioncermimujeres.es/sites/default/files/derechos_humanos_ de_las_mujeres_y_ninas.no8_.pdf

Comité sobre los derechos de las personas con discapacidad de Naciones Unidas (2018). Observación general número 6 sobre la igualdad y la no discriminación. Naciones Unidas: Convención sobre los derechos de las Personas con Discapacidad. https://www.cnlse.es/en/resources/legislaci\%C3\%B3n-internacional/2018observaci\%C3\%B3n-general-n\%C3\%BAm-6-2018-sobre-la-igualdad-y-la-no

Consejería de familia e igualdad de oportunidades de Castilla y León (2017). Acuerdo 7/17 del 9 de febrero de la Junta de Castilla y León, por el que se aprueba el plan estratégico de igualdad de oportunidades para las personas con discapacidad 2016/2020. Boletín Oficial de Castilla y León BOCYL, 29. 4189-4268.

Costa, A. P. (2016). Cloud Computing em Investigação Qualitativa: Investigação Colaborativa através do software webQDA. Fronteiras: Journal of Social, Technological and Environmental Science, 5(2), 153-161. https://doi.org/10.21664/2238-8869.2016v5i2.p153-161.

Sánchez-Gómez, M.C., \& Martín-Cilleros, M.V. (2017). Implementation of focus group in health research. Studies in Systems, Decision and Control, 71, pp. 49-61.

Gomiz, P. (2017). Vulnerabilidad y exclusión social en las mujeres con discapacidad. Documentación social, 184, 153-178.

Instituto Nacional de Estadística, INE (2009). Panorámica de la discapacidad en España. Encuesta de Discapacidad, Autonomía personal y situaciones de dependencia, 2008. Boletín Informativo del Instituto Nacional de Estadística, 1-12. https://www.ine.es/revistas/cifraine/1009.pdf

Martín-Cilleros, M.V., Navarro-Yuste, Y., \& Sánchez-Gómez, M.C. (2017). Experiencias y necesidades percibidas por las familias con un hijo con enfermedad rara, síndrome de CACH. Revista Psicologia, Diversidade e Saúde, 6(4), 286-299.

Martín-Cilleros, M.V., Sánchez-Gómez, M.C., Verdugo-Alonso, M.A., Crespo-Cuadrado, M., Sánchez-García, A.B., Caballo-Escribano, C., Verdugo-Castro, S., \& ManjónGarcía, E. (2020). En L. Fornari, F. Freitas, E. S. Fernandes de Oliveira, Cl. Oliveira, \& A. P. Costa (Eds.). Mujer y discapacidad: percepción de su calidad de vida. Investigaçao qualitativa em saúde: Avanços e Desafíos, (pp. 623-634). Ludomedia. https://doi.org/10.36367/ntqr.3.2020.623-634

Observatorio Estatal de la Discapacidad OED (2018). Personas con discapacidad y discriminación múltiple. Recuperado 14 octubre 2019: https://observatoriodeladiscapacidad.info/attachments/article/102/ DISCRIMINACION\%20MULTIPLE\%20OED.pdf 
Palacios, B., Gutiérrez, A., \& Sánchez, M. C. (2013). NVIVO 10: Una herramienta para la investigación en Comunicación. En M. Pacheco, M. Vicente, \& T. González (Eds.). Investigar la Comunicación hoy. Revisión de políticas científicas y aportaciones metodológicas. Simposio Internacional sobre Política Científica en Comunicación (Vol. 4, pp. 1003-1018).

Reis, L. P., \& Costa, A. P. (2017). Vantagens e desvantagens do uso de software na análise de dados qualitativos. RISTI - Revista Ibérica de Sistemas e Tecnologias de Informação, 23, ix-xiii. https://doi.org/10.17013/risti.23.o

Sánchez-Gómez, M.C., Delgado; M.C., \& Santos, M.C. (2013). El proceso de la investigación cualitativa. Manual de procedimiento: ejemplificación con una tesis doctoral. Edintras.

Sánchez-Gómez, M.C., Martín-Cilleros, M.V., Costa, A.P., \& y García-Peñalvo, F. (2018). Posicionamiento de la investigación en Ciencias Sociales. RISTI-Revista Ibérica de Sistemas e Tecnologias de Informação, 28, 102-113.

Sánchez Gómez, M. C., Palacios Vicario, B., \& Martín García, A. (2015). Indicadores de violencia de género en las relaciones amorosas. Estudio de caso en adolescentes chilenos. Pedagogía Social. Revista Interuniversitaria, 26, 85-109. DOI:10.7179/ PSRI_2015.26.04

Schalock, R. L., \& Verdugo, M. A. (2007). Calidad de vida: manual para profesionales de la educación, salud y servicios sociales. Alianza.

Shum, G., \& Conde, A. (2009). Género y discapacidad como moduladores de la identidad. Feminismos, 13, 119-132.

Souza, F. N., Costa, A. P., \& Moreira, A. (2011). Análise de Dados Qualitativos Suportada pelo Software WebQDA. Atas da VII Conferência Internacional de TIC na Educação: Perspetivas de Inovação (CHALLANGES2011), 49-56. 